\title{
PROGRAMA EDUCATIVO SOBRE ÚLCERA POR PRESSÃO COM EQUIPES DE ENFERMAGEM
}

\author{
BARON, Miriam Viviane ${ }^{1 *} ;$ GAYA, Anelise Reis ${ }^{2 * *}$; KRUG, Suzane Beatriz Frantz ${ }^{1 * *}$ \\ ${ }^{1}$ Universidade de Santa Cruz do Sul \\ ${ }^{2}$ Universidade Federal do Rio Grande do Sul \\ miriamvbaron@yahoo.com.br* \\ anegaya@gmail.com** \\ skrug@unisc.br ${ }^{* * *}$
}

\section{RESUMO}

Descrevem-se os principais aspectos vivenciados durante o desenvolvimento de programa educativo sobre úlceras por pressão com equipes de enfermagem atuantes em duas unidades de terapia intensiva do interior do Rio Grande do Sul. Trata-se de um relato de experiência. Os dados e informações referem-se ao período entre maio e agosto de 2014. Descrevem-se questões institucionais e pessoais envolvidas durante a realização do programa educativo. Os aspectos negativos relacionam-se à demanda de trabalho, ao espaço físico. A temática, a metodologia e as estratégias empregadas evidenciam motivação e interesse pelo aperfeiçoamento e ótima adesão, revelando feedback da percepção favorável como sendo aspectos positivos. Constatam-se obstáculos institucionais e pessoais que podem comprometer a qualidade assistencial pertinente à prevenção das úlceras por pressão. Sendo assim, sugere-se compromisso compartilhado entre equipe interdisciplinar e instituições para superar as barreiras na assistência. à realização do programa educativo durante o expediente $e$

PALAVRAS-CHAVE: Úlcera por pressão. Educação continuada. Equipe de Enfermagem. Fisioterapeutas.

\section{EDUCATIONAL PROGRAM ON PRESSURE ULCER WITH NURSING TEAMS}

\section{ABSTRACT}

The main aspects experienced during the development of an educational program on pressure ulcers with nursing teams working in two intensive care units in the interior of Rio Grande do Sul are described. This is an experience report. The data and information reported refer to the period between May and August 2014. Institutional and personal issues involved during the implementation of the educational program are described. The negative aspects are related to the demand for work, to the realization of the educational program during the working day and to the physical space. The thematic, methodology and strategies used show motivation and interest for improvement and good adherence, revealing positive perception feedback as positive aspects. Institutional and personal obstacles that may compromise the quality of care pertinent to the prevention of pressure ulcers are noted. Thus, it is suggested a shared commitment between interdisciplinary team and institutions to overcome the barriers in the assistance. KEYWORDS: Pressure ulcer. Education continuing. Nursing team. Physical therapists.

\section{PROGRAMA EDUCATIVO SOBRE LAS ÚLCERAS POR PRESIÓN CON EQUIPO DE ENFERMERÍA}

\section{RESUMEN}

Se describen los principales aspectos vivenciados durante el desarrollo de programa educativo sobre úlceras por presión con equipos de enfermería actuantes en dos unidades de terapia intensiva del interior de Rio Grande do Sul. Se trata de un relato de experiencia. Los datos e informaciones se refieren al período entre mayo y agosto de 2014. Como resultados, se describen cuestiones institucionales y personales involucradas durante la realización del programa educativo. Los aspectos negativos se relacionan con la demanda de trabajo, la realización del programa educativo durante el horario y el espacio físico. La temática, la metodología y las estrategias empleadas evidencian motivación e interés por el perfeccionamiento y óptima adhesión, revelando retroalimentación de la percepción favorable como siendo aspectos positivos. Se constatan obstáculos institucionales y personales que pueden comprometer la calidad asistencial pertinente a la prevención de las úlceras por presión. Siendo así, se sugiere compromiso compartido entre equipo interdisciplinario e instituciones para superar las barreras en la asistencia.

PALABRAS CLAVE: Úlcera por presión. Educación continua. Personal de Enfermería. Fisioterapeutas. 


\section{INTRODUÇÃO}

Atualmente observa-se uma transição demográfica e epidemiológica que contribui para um incremento relativo das doenças crônicas, implicando no aumento da utilização dos serviços de saúde (MENDES, 2010). Consequentemente, aumentam as internações hospitalares assim como o tempo de internação, muitas vezes requerendo cuidados complexos com incremento tecnológico e terapêutico em Unidade de Terapia Intensiva (UTI) (CASTRO; ARANAZ-ANDRÉS, 2010; MENDES, 2010). Os pacientes internados na UTI, geralmente, estão em grave estado de saúde e se demonstram instáveis com disfunções orgânicas, precisando de cuidados específicos da equipe multiprofissional de saúde (BACKES et al., 2012). Frequentemente se encontram acamados e debilitados e são susceptíveis a eventos adversos, em particular, a úlcera por pressão (UP) (BACKES et al., 2012; BRASIL, 2014).

No âmbito da UTI, as expectativas dos pacientes e familiares são focadas na melhora e cura dos problemas de saúde, e não no desenvolvimento de novas complicações durante a internação. Neste contexto, o desenvolvimento da UP no campo hospitalar é indicador de má qualidade da assistência prestada e enseja desconforto e desconfiança em relação às equipes assistenciais e instituição. Além desse fato, a presença da UP causa implicação psíquica, física e financeira, demanda gastos adicionais à instituição, exigindo tratamentos e medicamentos complementares, podendo também, contribuir com desfechos negativos na qualidade de vida e mortalidade (MOREHEAD; BLAIN, 2014; SILVA, A. et al., 2013).

As taxas de incidência de UP em UTIs no Brasil mantêm-se altas e variam de 22,2\% a 59,5\%, dependendo da região do país e da especialidade da UTI (BAVARESCO; MEDEIROS; LUCENA, 2011; SILVA, M. et al., 2013). Ademais, a cultura da qualidade na assistência ainda não está bem estabelecida por parte das instituições hospitalares, seja por desconhecimento, falta de recursos humanos qualificados, escassez de inovação e tecnologias de qualidade, entre outros aspectos (BUSSOTI et al., 2013; SILVA, R. et al., 2013).

A vulnerabilidade desse segmento populacional indica a premência de UTIs especializadas e de equipe multiprofissional capacitada, especialmente as equipes de enfermagem e fisioterapia, que prestam atendimento direto e contínuo aos pacientes, em um contexto no qual deve predominar o compromisso compartilhado entre profissionais de saúde no 
intuito de alcançar assistência com qualidade e segurança (BUSSOTI et al., 2013; MIYAZAKI; CALIRI; SANTOS, 2010). Neste domínio, estudos recentes mostram que programas educativos baseados em evidências científicas contribuem para o aumento do conhecimento das equipes que prestam atendimento contínuo ao paciente e visam diminuir a incidência da UP nas instituições hospitalares (MIYAZAKI; CALIRI; SANTOS, 2010; MOHAMMAD; JAMAL, 2012).

Surge, neste cenário, a necessidade de busca de estratégias que impulsionem transformações, e a Educação Continuada (EC) apresenta-se como meio propulsor, que permite a criação de oportunidades de capacitação e desenvolvimento interpessoal e interprofissional, considerando as necessidades das equipes, da instituição e do cliente, com o intuito de proporcionar atenção à saúde com qualidade (BEZERRA et al., 2012).

Buscando contribuir com o conhecimento nesta área, o presente estudo teve como objetivo descrever os principais aspectos vivenciados pela pesquisadora durante o desenvolvimento de um programa educativo sobre UP, realizado com equipes de enfermagem atuantes em UTI adulto.

\section{MÉTODO}

Trata-se de um estudo descritivo, tipo relato de experiência, no decurso do desenvolvimento de um programa educativo com equipes de enfermagem atuantes em duas UTIs adulto do interior do Rio Grande do Sul. Trata-se de dois hospitais de médio porte: um hospital dispõe de UTI com dez leitos e outro hospital possui UTI com sete leitos. As equipes de enfermagem das duas UTIs compunham um total de 50 funcionários. Destes, oito eram enfermeiros e 42 eram técnicos de enfermagem. Os profissionais atuavam nas UTIs nos turnos da manhã, tarde e noite. Os dados e informações referem-se ao período de pesquisa que aconteceu entre maio e agosto de 2014.

Após a autorização das instituições hospitalares para a realização da pesquisa e aprovação pelo Comitê de Ética em Pesquisa da Universidade de Santa Cruz do Sul, com protocolo no 639.428 e CAAE: 19329014.0.0000.5343, deu-se início à investigação.

Primeiramente foi realizado um estudo piloto com os profissionais, no qual foi entregue o instrumento composto por itens referentes a dados sociodemográficos dos participantes e teste 
de conhecimento específico sobre UP para mensurar o tempo de preenchimento e esclarecer dúvidas sobre a compreensão do mesmo. Num segundo momento foi entregue o instrumento composto pelos dados sociodemográficos e teste de conhecimento que visou mensurar o nível de conhecimento sobre estadiamento, avaliação e prevenção da UP dos profissionais. Para tal, aplicou-se o Teste de Conhecimento de Pieper, validado para a utilização com equipes de enfermagem em estudo anterior realizado no Brasil (FERNANDES; CALIRI; HAAS, 2008). O teste foi aplicado antes do início e ao término do programa educativo. Este teste é validado para o emprego com profissionais de enfermagem atuantes em UTI e é baseado nas recomendações propostas por diretrizes internacionais. É constituído por 41 afirmações verdadeiras ou falsas, com oito questões sobre estadiamento e avaliação da UP e 33 acerca da prevenção da UP. Para cada uma das propostas, o participante deveria selecionar uma resposta, considerando as opções verdadeiro (V), falso (F) e não sei (NS). Para cada acerto foi atribuído um ponto. Para as respostas erradas ou para aquelas respondidas como "não sei", o escore atribuído foi zero. O escore total do teste de conhecimento correspondeu à soma de todas as respostas corretas. Conforme os autores do teste, o nível de conhecimento do participante era considerado adequado quando este obtivesse $90 \%$ ou mais de acertos no teste, e a questão era considerada conhecida quando $90 \%$ ou mais dos participantes a respondessem corretamente (PIEPER; MOTT, 1995).

A coleta de dados e o programa educativo foram realizados pela pesquisadora principal. O instrumento foi distribuído aos sujeitos, que o responderam individual e imediatamente, durante o horário de trabalho e o devolveram à pesquisadora dentro de um envelope sem identificação, garantindo o anonimato dos participantes. Todos os profissionais de enfermagem atuantes nas UTIs que não estavam de férias no momento da aplicação do instrumento inicial e que assinaram o Termo de Consentimento Livre e Esclarecido (TCLE) participaram do estudo, compondo um quadro total de 50 profissionais participantes na pesquisa.

Após a aplicação do instrumento, deu-se início ao programa educativo com as equipes. 0 cronograma do programa educativo foi disposto de maneira a ser realizado uma vez por semana, por dez semanas, totalizando dez encontros de uma hora de duração. Este foi realizado em salas nas UTIs, na forma de Workshop, com apresentação de slides em power point, discussão em grupo, prática de posicionamentos e entrega de slides impressos. Foram abordados a descrição geral e as recomendações sobre prevenção da UP com base em diretrizes internacionais (EPUAP; NPUAP, 
2009) e publicações científicas atuais sobre o assunto. Posteriormente à finalização do programa educativo foi reaplicado o teste de conhecimento para avaliar o nível de conhecimento das equipes de enfermagem. Os dados foram sumarizados e analisados segundo a estatística descritiva.

O presente relato de experiência baseou-se nas informações descritas pela pesquisadora principal em diário de campo. O diário foi elaborado durante a pesquisa de campo e consta da observação e narração dos fatos e fenômenos ocorridos durante as etapas de desenvolvimento do programa educativo com as equipes de enfermagem atuantes nas UTIs.

Vale acrescentar que o presente relato de experiência foi vivenciado pela pesquisadora, profissional de enfermagem e de fisioterapia, durante a etapa da coleta de dados de pesquisa, e fez parte da dissertação de mestrado do programa de Pós-Graduação/Mestrado em Promoção da Saúde da Universidade de Santa Cruz do Sul (Unisc), intitulada Estratégia de intervenção educativa sobre úlcera por pressão: estudo com equipes de enfermagem de Unidades de Tratamento Intensivo de hospitais dos Vales do Rio Pardo e Jacuí/RS, apresentada ao programa de Pós-Graduação da referida instituição em fevereiro de 2015.

\section{RESULTADOS}

O espaço físico das UTIs onde o programa educativo foi realizado não dispunha de sala para atividades educacionais. As salas específicas para encontros e palestras com acomodação e multimídia localizavam-se fora e distantes das UTIs. As coordenadoras de enfermagem sinalizaram a inviabilidade do deslocamento dos profissionais para fora das UTIs durante a jornada de trabalho, para a participação no programa educativo. Estas justificaram o fato devido à acentuada demanda de trabalho, e à necessidade de cuidados complexos e contínuos com os pacientes deste setor. Neste sentido, foi acordado com as coordenadoras e equipes de enfermagem que as intervenções seriam realizadas em sala disponível e com possibilidade de acomodação e privacidade dentro das próprias UTIs. Para esta finalidade, utilizou-se da sala de recepção de familiares e da sala de coordenação da enfermagem localizadas dentro das UTIs, conforme a disponibilidade destas. Para a apresentação do conteúdo programático, disponibilizou-se de computador próprio e, para a execução das atividades práticas, foi disponibilizado leito das próprias UTIs.

Educação \& Formação, Fortaleza, v. 3, n. 7, p. 124-136, jan./abr. 2018

DOI: http://dx.doi.org/ 
Conforme o cronograma da pesquisa, o programa educativo, em forma de Workshop, seria realizado em dez encontros com as equipes de enfermagem de cada UTI. Estes aconteceriam uma vez por semana, por dez semanas, totalizando dez encontros de uma hora. Contudo, vários profissionais possuíam atividades complementares, como duplo emprego, ou cursavam ensino superior. Além disso, alguns profissionais residiam em localidades afastadas do local de trabalho e não dispunham de transporte público. Ademais, os gerentes institucionais solicitaram que o programa ocorresse durante o expediente de trabalho, em razão da inviabilidade institucional de manter o quadro em jornada de trabalho extraordinário e o acréscimo financeiro decorrente desta. Estes fatores justificaram a necessidade da realização dos encontros durante o expediente de trabalho. Foi acordado com as equipes de enfermagem que os encontros ocorreriam durante o horário de trabalho, no decorrer dos turnos da manhã, tarde e noite.

Neste complexo ambiente, buscou-se evitar prejuízos à rotina de trabalho dos participantes da pesquisa e dos pacientes. Assim, a pesquisadora fez-se presente no setor da UTI das $5 \mathrm{~h} 30$ às $22 \mathrm{~h}$, conforme a necessidade, atendendo os turnos diurno e noturno. Aguardava-se o melhor momento para realizar o programa educativo, o que era definido pelos próprios profissionais de enfermagem das UTIs, considerando a demanda de trabalho diária. Geralmente, o programa educativo era realizado com duplas de trabalhadores, haja vista a necessidade de afastamento do posto de enfermagem por determinado período, o que contribuía para o aumento de encargos e responsabilidades sobre os demais colegas. Quando não era possível abordar algum participante em razão de folga, atestado, remanejo para outro setor, ou ainda em situações de grande demanda de trabalho, a pesquisadora retornava no plantão seguinte e, quando necessário, nos finais de semana. Dessa forma, houve a necessidade de realização de cento e quatro encontros educativos.

Durante o programa educativo foram abordadas questões pertinentes ao desenvolvimento e prevenção da UP como anatomia e fisiologia da pele, definição e estágios da UP, etiopatogenia, custos, avaliação do risco, avaliação da pele, nutrição, reposicionamentos e superfícies especiais de apoio para a prevenção. O conteúdo programático foi desenvolvido com base em artigos científicos atuais e em diretrizes internacionais para a prevenção da UP.

O programa educativo foi realizado em forma de Workshop, no qual os participantes receberam conteúdo impresso, assistiram à apresentação de slides e participaram ativamente 
das discussões, em um diálogo aberto e com troca de ideias, assim como das atividades práticas. Os participantes expuseram situações da prática e da experiência profissionais, compartilhando vivências, nas quais se buscou conciliar o conhecimento da teoria com a prática, diante de embasamento teórico pertinente, e buscando as melhores evidências científicas disponíveis sobre $o$ assunto.

Dentre os aspectos positivos no decorrer da pesquisa, aponta-se a cordial receptividade da pesquisadora pelos gestores e equipes de enfermagem durante a coleta de dados, o bom relacionamento, a dedicação, o comprometimento e a empatia recíproca. A citação deste programa educativo pelos profissionais de enfermagem como ponto positivo em uma pesquisa de clima organizacional realizado em uma UTI e o estímulo, por parte dos profissionais, para a criação de um protótipo de prevenção da UP em calcâneo também podem ser interpretados como aspectos satisfatórios. Destaca-se a ausência de desistências no decorrer do programa, pois, mesmo em período de férias, os profissionais participaram do programa educativo semanalmente - desta forma, mantendo o cronograma proposto. Ao final do programa, todos os participantes receberam certificado de participação, sendo esta uma forma de valorização das equipes que foram fundamentais para a concretização do estudo.

Os aspectos negativos se referem à falta de sala adequada para a realização do programa educativo, as longas esperas pelos profissionais para a realização dos encontros educativos, a necessidade de retorno em dia posterior para abordar os ausentes e o elevado número de encontros com as equipes de enfermagem.

\section{DISCUSSÃO}

O programa educativo aconteceu na esfera das UTIs. O setor da UTI é composto de estrutura física, recursos humanos, materiais e tecnologias para o tratamento intensivo de pacientes graves. O ambiente compreende a equipe multiprofissional, que é composta por enfermeiros, técnicos de enfermagem, fisioterapeutas, médicos, nutricionistas, psicólogos e farmacêuticos, e estes devem realizar cuidado interdisciplinar e humanizado com foco na recuperação da saúde dos doentes. Conforme estudiosos sobre o assunto, é neste complexo cenário que atividades educativas devem permear o cotidiano de trabalho dos profissionais da 
saúde, sendo fundamental seu início durante o período de formação, subsistindo ao longo do exercício profissional por meio da EC (BUSSOTI et al., 2013). A EC, por sua vez, visa à atualização e ao aperfeiçoamento dos profissionais, com vistas à melhoria na qualidade do atendimento e à excelência na assistência (BUSSOTI et al., 2013; RODRIGUEZ et al., 2011).

Destaca-se a falta de espaço físico e recursos didáticos, uma vez que as atividades deveriam ser realizadas dentro das UTIs, tornando-se de suma importância a disposição de local físico específico na unidade. Este espaço real pode contribuir de forma a estimular a EC nos profissionais de saúde, focando em questões pertinentes elegidas pelos profissionais e gerenciadas pela equipe interdisciplinar da unidade, atentando para a responsabilidade dos profissionais de saúde no processo ensino-aprendizagem, principalmente em instituições em que a EC ainda não está bem estabelecida. Um pesquisador sobre o assunto sugere a necessidade de salas apropriadas, com material didático e biblioteca dentro da UTI para que ocorra de forma concreta o processo da EC neste setor (BEZERRA et al., 2012).

Com relação ao elevado número de encontros (total de cento e quatro), justificado pelo fato destes ocorrerem em duplas e devido à necessidade de retorno da pesquisadora em dias subsequentes para abordar todos, leva a refletir sobre a complexidade da EC no ambiente da UTI. Em estudo recente, uma pesquisadora relata que as dificuldades para o desenvolvimento de programas de EC no âmbito hospitalar estão relacionadas, particularmente, à falta de disponibilidade durante o plantão para a realização do programa por demanda de serviço e à ausência de espaço para a realização das intervenções, demonstrando que a EC é um processo prolongado que vai além dos sistemas educacionais (ROGENSKI; KURCGANT, 2012).

Um estudo realizado em um hospital universitário no Brasil apontou que metade dos enfermeiros investigados tinha dificuldade para participar de programas de EC. Este fato se devia ao horário em que as atividades eram realizadas, por possuírem os enfermeiros duplo vínculo empregatício; em virtude de carga horária exaustiva, demanda do serviço, falta de pessoal para cobertura no setor, domicílio em outra cidade, por problemas familiares, pouco reconhecimento da profissão e coincidência com outros eventos. Estas razões, associadas à jornada doméstica, levaram o pesquisador do estudo a inferir que os programas de EC devem ser realizados durante o expediente de trabalho (BEZERRA et al., 2012).

Educação \& Formação, Fortaleza, v. 3, n. 7, p. 124-136, jan./abr. 2018 
O conteúdo programático do programa educativo foi desenvolvido com base em artigos científicos atuais e nas diretrizes internacionais de prevenção da UP do European Pressure Ulcer Advisory Panel e do National Pressure Ulcer Advisory Panel (EPUAP/NPUAP), que fornecem recomendações baseadas em evidências científicas atuais e estão disponíveis para todos os países, sendo adequadas também para o cenário brasileiro, visto que não existem normativas e diretrizes para a prevenção da UP no Brasil (EPUAP; NPUAP, 2009; ROGENSKI; KURCGANT, 2012).

O programa educativo foi realizado em forma de Workshop e teve participação dinâmica dos participantes nas discussões e atividades práticas. Desta forma, os participantes foram envolvidos em um processo de interação com o ambiente, a teoria e a prática, assimilando novos conteúdos e informações que se relacionam ao saber em curso, integrando um processo psicológico interno de elaboração e aquisição de aprendizagens (KNUD, 2013).

Além disso, os participantes expuseram situações da prática profissional, compartilhando vivências, no qual se buscou confrontar o conhecimento empírico com as melhores evidências científicas disponíveis sobre o assunto. A prática baseada em evidências é uma forma sistemática mediante a qual se visa selecionar literatura científica relevante, avaliar as provas e decidir sobre a sua aplicação em um dado problema ou situação, com base na melhor evidência atual, evitando práticas obsoletas e baseadas em ações arbitrárias (BARRIA-PAILAQUILEN, 2013). Ademais, a busca pelo conhecimento revela o interesse pelo autodesenvolvimento, o que é oportuno para o aperfeiçoamento da equipe de trabalho, a segurança do paciente e a valorização profissional (BEZERRA et al., 2012).

Dentre os aspectos negativos, no presente estudo constataram-se obstáculos institucionais e pessoais como a grande demanda de trabalho diária e a falta de profissionais extras para suprir a equipe durante a realização do programa educativo no decurso da jornada de trabalho, contribuindo para este fato a necessidade de realização das atividades educativas durante o expediente, a inviabilidade de jornada extraordinária, além de duplo vínculo empregatício de alguns profissionais. Este contexto mostra a complexidade referente ao desenvolvimento da EC no âmbito da UTI, onde a disponibilidade de profissionais capacitados é indissociável de conhecimento atual, baseado em evidências, em vistas de uma assistência segura e de qualidade. Em um estudo atual, um investigador levantou sugestões das equipes de saúde para a melhoria do processo de EC. Entre estas foi recomendada a realização do programa 
educativo durante o expediente de trabalho, com disponibilização de sala apropriada com material didático e, principalmente, menor demanda de serviço para poder participar dos encontros (BEZERRA et al., 2012).

Dentre os aspectos positivos, no decorrer da pesquisa, pode-se citar o bom relacionamento entre a pesquisadora e as equipes de enfermagem, o que pode ter contribuído para o engajamento dos profissionais na participação do programa educativo e na ausência de desistências durante o processo. Um investigador sobre o assunto assevera que fatores como o bom relacionamento entre pesquisador e profissionais de enfermagem, bem como a adesão ao programa e a vontade demonstrada pelos profissionais no aprimoramento do conhecimento, facilitam o desenvolvimento de programas de EC (RODRIGUEZ et al., 2011).

A despeito das prolongadas esperas por parte da pesquisadora ser um aspecto negativo nesta pesquisa, estas proporcionaram longos períodos de convívio semanal e favoreceram vínculos positivos entre ela e as equipes. Acredita-se que os aspectos positivos mencionados nesta pesquisa contribuíram para superar os aspectos negativos e as adversidades, permitindo o processo ensino-aprendizagem. Neste estudo, não houve avaliação da opinião dos participantes sobre o programa educativo, o que se considera uma limitação. Todavia, um aspecto marcante a ser evidenciado é o feedback da percepção favorável, bem como o interesse pelo aperfeiçoamento por parte dos participantes, que se mostraram motivados e com um índice de $100 \%$ de adesão ao programa, corroborando os resultados encontrados em estudo do gênero que utilizou o processo de feedback na avaliação da intervenção e do programa de EC e observou impacto positivo para o desempenho e qualificação profissional (RODRIGUEZ et al., 2011).

Além disso, como efeito positivo deste programa educativo, podemos citar a diminuição da incidência de UP em uma UTI. Foram analisados os dados de incidência de UP em uma UTI quatro meses antes do início do programa educativo e constatou-se incidência de 17,2\% de UP. Após quatro meses da intervenção educativa, foi realizada nova análise de incidência na mesma UTI e verificou-se incidência de $14,2 \%$ de UP. Os resultados desta pesquisa são similares aos resultados de um estudo realizado em UTI no Brasil, em que as pesquisadoras constataram redução da incidência de UP após a instituição de um protocolo de avaliação de risco e prevenção da UP (ROGENSKI; KURCGANT, 2012).

Educação \& Formação, Fortaleza, v. 3, n. 7, p. 124-136, jan./abr. 2018

DOI: http://dx.doi.org/ 
Ademais, os participantes deste estudo, na vivência do contexto da UTI, destacaram a necessidade de um dispositivo para a prevenção da UP em calcâneo e solicitaram o desenvolvimento de um produto efetivo para este problema, sendo este contexto interdisciplinar, congruente para o desenvolvimento de novas tecnologias consideradas relevantes e oportunas neste âmbito.

Fatores limitadores neste estudo foram a ausência de um instrumento validado de conhecimento sobre a prevenção da UP para a utilização com a equipe multiprofissional. A origem da UP é multifatorial e a sua prevenção deve ser efetivada através de abordagem interdisciplinar, especialmente com a participação do fisioterapeuta, que atua no atendimento direto ao paciente na UTI de dezoito a vinte e quatro horas diárias; um dos principais objetivos de sua atuação é a mobilização passiva e ativa dos pacientes. O fisioterapeuta é corresponsável pela prevenção da UP, principalmente na mudança de decúbito, assim como no correto alinhamento corporal e conforto do paciente acamado, necessário para proporcionar o alívio da dor oriunda de posturas inadequadas no leito, e no ensinamento de técnicas para aliviar a pressão autonomamente, quando este estiver acordado. Além de que, o fisioterapeuta deve integrar os programas de EC conjuntamente com a equipe de enfermagem e os demais profissionais da saúde e participar na elaboração e instituição de bundles de mobilização e prevenção da UP, no intuito de evitar este fatídico problema, visto que a prevenção pode efetivar-se com a adoção de medidas simples e do compromisso compartilhado.

\section{CONSIDERAÇÕES FINAIS}

A temática abordada por intermédio desta metodologia e as estratégias empregadas evidenciaram a motivação e o interesse pelo aperfeiçoamento do conhecimento, mostrando ótima adesão ao programa, assim como revelando feedback da percepção favorável por parte dos profissionais. Houve diminuição da incidência da UP em uma UTI após o programa educativo. Assim sendo, acredita-se que houve aprimoramento do conhecimento dos participantes acerca do tema. Além disso, o bom relacionamento e a interação interdisciplinar suscitaram a necessidade do desenvolvimento de novas tecnologias consideradas necessárias neste âmbito.

Educação \& Formação, Fortaleza, v. 3, n. 7, p. 124-136, jan./abr. 2018

DOI: http://dx.doi.org/

http://seer.uece.br/redufor 
A falta de espaço físico específico para o desenvolvimento do programa educativo, de recursos didáticos, o elevado número de encontros devido à grande demanda de serviço diário, a falta de profissionais extra para suprir a equipe durante a atividade educativa, a realização do programa durante o expediente, a inviabilidade de jornada extraordinária e o duplo vínculo empregatício de alguns profissionais evidenciam que o processo de EC é complexo e vai além dos sistemas educacionais.

Acredita-se que para vencer estas barreiras é necessário o compromisso compartilhado entre profissionais de saúde e instituições, e que a cultura de qualidade da assistência deve ser parte integrante da missão das instituições hospitalares.

\section{REFERÊNCIAS}

BACKES, M. T. S. et al. O cuidado intensivo oferecido ao paciente no ambiente de unidade de terapia intensiva. Escola Anna Nery, Rio de Janeiro, v. 16, n. 4, p. 689-696, 2012.

BARRIA-PAILAQUILEN, R. M. Practice based on evidence: An opportunity for quality care. Investigación y Educación en Enfermería, Colombia, v. 31, n. 2, p. 181-182, 2013.

BAVARESCO, T.; MEDEIROS, R. H.; LUCENA, A. F. Implantação da escala de Braden em uma unidade de terapia intensiva de um hospital universitário. Revista Gaúcha de Enfermagem, Porto Alegre, v. 32, n. 4, p. 703-710, 2011.

BEZERRA, A. L. Q. et al. O processo de educação continuada na visão de enfermeiros de um hospital universitário. Revista Eletrônica de Enfermagem, Goiânia, v. 14, n. 3, p. 618-625, 2012.

BRASIL. Ministério da Saúde. Agência Nacional de Vigilância Sanitária. Fundação Oswaldo Cruz. Anexo 02: Protocolo para prevenção de úlcera por pressão. Brasilia, DF: Ministério da Saúde, 2014.

BUSSOTI, A. et al. Programa educacional para unidades de terapia intensiva neonatais e pediátricas brasileiras. Trabalho, Educação e Saúde, Manguinhos, v. 11, n. 3, p. 611-631, 2013.

CASTRO, M. T. G. V.; ARANAZ-ANDRÉS, J. M. Adverse events associated with the healthcare of patients with multimorbidity admitted to medium-long stay hospitals. Medicina Clínica, Barcelona, n. 1, p. 17-23, 2010.

EUROPEAN PRESSURE ULCER ADVISORY PANEL AND NATIONAL PRESSURE ULCER ADVISORY PANEL (EPUAP-NPUAP). Prevention and treatment of pressure ulcers: Quick reference guide. Washington, DC: EPUAP, 2009. 
FERNANDES, L. M.; CALIRI, M. H. L.; HAAS, V. J. Efeito de intervenções educativas no conhecimento dos profissionais de enfermagem sobre prevenção de úlceras por pressão. Acta Paulista de Enfermagem, São Paulo, v. 21, n. 2, p. 305-311, 2008.

KNUD, I. Teorias contemporâneas da aprendizagem. Porto Alegre: Penso, 2013.

MENDES, E. V. As redes de atenção à saúde. Ciência e Saúde Coletiva, Rio de Janeiro, v. 15, n. 5, p. 2297-2305, 2010.

MIYAZAKI, M. Y.; CALIRI, M. H. L; SANTOS, C. B. Knowledge on Pressure ulcer prevention among nursing professionals. Revista Latino-Americana de Enfermagem, São Paulo, v. 18, n. 6, p. 12031211, 2010.

MOHAMMAD, Y. N. S.; JAMAL, A. M. S. Q.; DENIS, A. An interventional study on the effects of pressure ulcer education on Jordanian registered nurses' knowledge and practice. Procedia Social and Behavioral Sciences, United States, v. 47, p. 2196-2206, 2012.

MOREHEAD, D.; BLAIN, B. Driving hospital-acquired pressure ulcers to zero. Critical Care Nursing Clinics of North America, United States, v. 26, n. 4, p. 559-567, 2014.

PIEPER, B.; MOTT, M. Nurses' Knowledge of pressure ulcer prevention, stating and description. Advances in Wound Care, Philadelphia, v. 8, n. 3, p. 34-48, 1995.

RODRIGUEZ, E. O. L. et al. Implantação de educação continuada com profissionais de enfermagem utilizando a pedagogia problematizadora: relato de experiência. Revista de Enfermagem do Centro Oeste Mineiro, Minas Gerais, v. 1, n. 4, p. 583-591, 2011.

ROGENSKI, N. M. B.; KURCGANT, P. The incidence of pressure ulcers after the implementation of a prevention protocol. Revista Latino-Americana de Enfermagem, São Paulo, v. 20, n. 2, p. 333339, 2012.

SILVA, A. J. et al. Economic cost of treating pressure ulcers: A theoretical approach. Revista da Escola de Enfermagem da USP, São Paulo, v. 47, n. 4, p. 971-976, 2013.

SILVA, M. L. N. et al. Úlcera por pressão em unidade de terapia intensiva: análise da incidência e lesões instaladas. Revista da Rede de Enfermagem do Nordeste, Fortaleza, v. 14, n. 5, p. 938-944, 2013.

SILVA, R. B. et al. Qualidade da assistência de enfermagem em unidade de terapia intensiva de um hospital escola. Revista Gaúcha de Enfermagem, Porto Alegre, v. 34, n. 4, p. 114-120, 2013.

Recebido em 17 de março de 2017.

Aceito em 20 de setembro de 2017.

Educação \& Formação, Fortaleza, v. 3, n. 7, p. 124-136, jan./abr. 2018

DOI: http://dx.doi.org/

http://seer.uece.br/redufor 